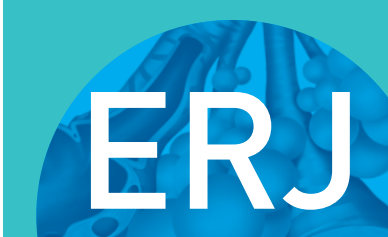

open research

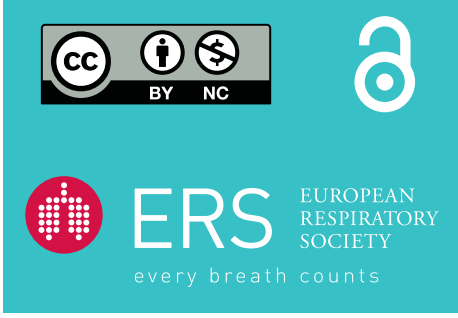

\section{Heightened response to e-cigarettes in COPD}

\section{To the Editor:}

E-cigarettes are used as an alternative to cigarette smoking, and as nicotine replacement therapy, with suggestions that they are markedly less harmful than cigarettes to the user. The confusion around the safety of e-cigarettes stems from contradictory findings in which variations in experimental methodology and the testing of different devices has not been accounted for. The harms associated with their use are not well understood and this is commonly misconceived as meaning that they are a healthy alternative to smoking. This misconception is further exacerbated by physicians and public health bodies which have made recommendations without strong scientific evidence [1]. Multiple studies have concluded that e-cigarette vapour exposure could lead to inflammation [2-5], emphysema [4] and a greater risk of bacterial and viral infection [3]. The lack of a defined model for e-cigarette exposure for both in vitro cellular and in vivo animal studies has led to contradictory findings between studies. Such differences can be attributed to different devices (first versus fourth generation), vaporisation temperature, different E-liquids or the concentration of e-cigarette vapour used. The confusion caused by contradictory findings leaves consumers and clinicians to form their own opinions about e-cigarettes safety which may lead to further public health issues in the future. Furthermore, no studies have compared responses in cells from people with chronic obstructive pulmonary disease (COPD), a disease state where the use of e-cigarettes is particularly attractive. However, COPD lung cells are known to be hyperresponsive to a range of environmental stimuli including cigarette smoke and pollution, and therefore might also respond differently to e-cigarette vapour.

The aim of this study was to evaluate dose-response relationships of e-cigarette stimulation of primary airway smooth muscle cells (ASMCs) from people with and without COPD under realistic physiological conditions. ASMCs were chosen for this study because of their contribution to pathological processes in COPD. Not only is smooth muscle bulk increased in COPD airways, ASMCs have been shown to secrete increased inflammatory mediators and chemokines compared with cells from smokers without COPD [6], suggesting their response to inflammatory stimuli might contribute to lung inflammation and/or disease progression in COPD. We have previously shown that ASMCs and airway fibroblasts from people with COPD are hyperresponsive to cigarette smoke [6,7], and hypothesised that they would also be hyperresponsive to e-cigarettes. We also hypothesised that the increased toxic by-product formation seen at higher vaporisation temperatures would result in greater cytotoxicity in ASMCs [8].

ASMCs from 22 patients were included in this study, of which nine had a diagnosis of COPD (forced expiratory volume in $1 \mathrm{~s}(\mathrm{FEV} 1)<80 \%, \mathrm{FEV}_{1} /$ forced vital capacity $\left.<0.7\right)$. The non-COPD patients had normal lung spirometry. E-cigarette vapour extract (EVE) was made by bubbling $20 \times 5 \mathrm{~s}$ bursts of E-vapour through $25 \mathrm{~mL}$ of DMEM supplemented with $0.1 \%$ Fetal Bovine Serum (FBS) (JRH Biosciences, Melbourne, Australia), $2.5 \mu \mathrm{g} \cdot \mathrm{mL}^{-1}$ amphotericin B, $20 \mu \mathrm{g} \cdot \mathrm{mL}^{-1}$ streptomycin and $20 \mathrm{U} \cdot \mathrm{mL}^{-1}$ penicillin (Invitrogen, Mount Waverly, Australia). CXCL8 release was measured using an ELISA kit (R\&D systems, Minneapolis, MN, USA) according to the manufacturer's instructions. Cell viability was assessed by measuring mitochondrial activity. $10 \mu \mathrm{L}$ of 3-(4,5-dimethylthiazol-2-yl)-2,5-diphenyltetrazolium bromide (MTT) dye (Sigma Aldrich, Castle Hill, Australia) $(0.005 \% \mathrm{w} / \mathrm{v}$ in sterile PBS) was added to each well, followed by incubation for $6 \mathrm{~h}$ at $37^{\circ} \mathrm{C}$ in $5 \%$ carbon dioxide.

@ERSpublications

E-cigarettes induce greater inflammatory mediators from COPD lung cells; therefore, the risks of e-cigarette use in COPD might be greater than in people without COPD http://ow.ly/ xmnN30nzDhX

Cite this article as: Bozier J, Rutting S, Xenaki D, et al. Heightened response to e-cigarettes in COPD. ERJ Open Res 2019; 5: 00192-2018 [https://doi.org/10.1183/23120541.00192-2018].

Copyright ( $\mathrm{ERS}$ 2019. This article is open access and distributed under the terms of the Creative Commons Attribution Non-Commercial Licence 4.0. 
To investigate if lung cells from people with COPD are hyperresponsive to e-cigarettes we stimulated COPD and non-COPD cells with increasing concentrations of tobacco- and menthol-flavoured EVEs containing $18 \mathrm{mg}$ nicotine $\cdot \mathrm{mL}^{-1}\left(\mathrm{n}=7\right.$ COPD, $\mathrm{n}=7$ non-COPD) or $0 \mathrm{mg}$ nicotine $\cdot \mathrm{mL}^{-1}$ ( $\mathrm{n}=9$ COPD, $\left.\mathrm{n}=6 \mathrm{non}-\mathrm{COPD}\right)$.
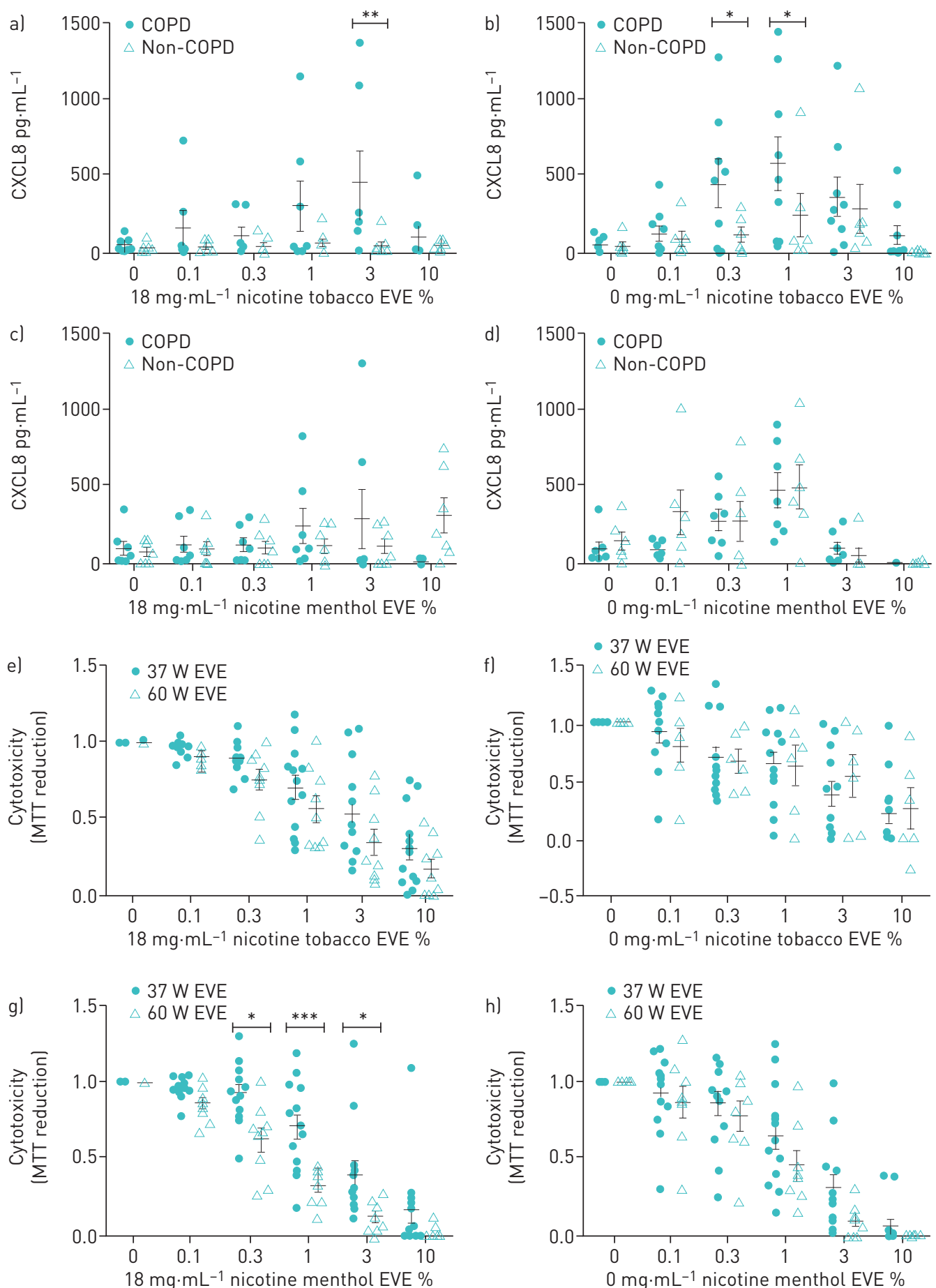

FIGURE 1 Primary human airway smooth muscle cells were stimulated with different concentrations of a, el $18 \mathrm{mg} \cdot \mathrm{mL}^{-1}$ nicotine tobacco-flavoured e-cigarette vapour extract (EVE), b, f) $0 \mathrm{mg} \cdot \mathrm{mL}^{-1}$ nicotine tobacco-flavoured EVE, c, g) $18 \mathrm{mg} \cdot \mathrm{mL}^{-1}$ nicotine menthol-flavoured EVE and $\left.\mathrm{d}, \mathrm{h}\right) 0 \mathrm{mg} \cdot \mathrm{mL}^{-1}$ nicotine menthol-flavoured EVE for $24 \mathrm{~h}$. The control well contained 0.1\% FBS DMEM. a-d) CXCL8 release production was measured in supernatant using ELISA. Error bars represent \SEM. Two-way ANOVA with Tukey's post-test was used for statistical analysis, significance is represented as *: $p \leqslant 0.05 ;{ }^{* *}: p \leqslant 0.01$. e-h) Cytotoxicity was determined post-stimulation using an MTT assay. Data are normalised to unstimulated cells and error bars represent \pm SEM. Two-way ANOVA with Tukey's post-test was used for statistical analysis, significance is represented as *: $p \leqslant 0.05 ; * * *: p \leqslant 0.001$. COPD: chronic obstructive pulmonary disease; MTT: 3-(4,5-dimethylthiazol-2-yl)-2,5-diphenyltetrazolium bromide. 
We selected these flavours as they proved to be more popular in smokers attempting to abstain from smoking [9]. Both tobacco EVEs induced greater CXCL8 production in COPD cells compared with non-COPD cells (figure 1a and b); however, this was not observed with the menthol EVEs (figure 1c and d). Next, we investigated the effects of vaporisation temperature on toxicity, and found that menthol EVE containing nicotine was more cytotoxic when generated at $60 \mathrm{~W}$, with a trend towards increased toxicity with menthol flavoured nicotine-free EVE (figure $1 \mathrm{~g}$ and $\mathrm{h}$ ). In contrast, tobacco-flavoured EVE had no effect on cytotoxicity when generated at higher temperatures (figure 1 e and f).

Cytotoxicity and inflammation are aetiological in COPD as they both contribute to pathological processes of the disease. Emphysema is caused by alveolar cell death and ineffective repair, resulting in an enlargement of the alveolar spaces [10]. The cytotoxicity of cigarette smoke is well documented and considered a contributing factor to disease progression. We have previously shown that cigarette smoke extract is cytotoxic to primary human ASMCs using methodology similar to that used with EVEs here [6]. This method is representative of what happens in the lung tissue, as only soluble components of the smoke or e-vapour will permeate through the epithelial layer to the underlying mesenchymal cells.

The power settings of the e-cigarette used were chosen according to the manufacturer's guidelines and users' preferences. The maximum power recommended for this coil $(60 \mathrm{~W})$ was used, as users often prefer to use their device at the high settings. Interestingly at the higher power setting, we found that that there was a significant increase in cytotoxicity with $18 \mathrm{mg}$ nicotine $\cdot \mathrm{mL}^{-1}$ menthol e-vapour extract. Coils were inspected before and after each extract generation and the tanks were refilled with e-liquid to confirm no dry wicking was taking place.

Patients with COPD, or smokers, might switch to e-cigarettes as an alternative nicotine source, believing that they are safer. All e-cigarette aerosols increased CXCL8 production in ASMCs irrespective of flavour or nicotine concentration. This suggests that e-cigarettes would stimulate lung neutrophilic inflammation. Furthermore, our data suggests that e-cigarette aerosol stimulates COPD cells in a similar manner to cigarette smoke, resulting in an increased production of CXCL8 from COPD cells compared with non-COPD cells.

Overall, our data suggests that COPD patients should avoid using e-cigarettes as a smoking cessation aid as they have a similar ability to stimulate inflammation and lung damage as cigarette smoke, and thus potentially accelerate their disease progression.

Jack Bozier $^{1,2}$, Sandra Rutting ${ }^{1}$, Dia Xenaki ${ }^{1}$, Matthew Peters ${ }^{3}$, Ian Adcock ${ }^{4,5}$ and Brian G. Oliver ${ }^{1,2}$ ${ }^{1}$ Respiratory Cellular and Molecular Biology, Woolcock Institute of Medical Research, University of Sydney, Sydney, Australia. ${ }^{2}$ School of Life Sciences, University of Technology Sydney, Sydney, Australia. ${ }^{3}$ Concord Hospital, University of Sydney, Sydney, Australia. ${ }^{4}$ Airway Diseases Section, National Heart and Lung Institute, Imperial College London, London, UK. ${ }^{5}$ Biomedical Research Unit; Section of Respiratory Diseases, Royal Brompton and Harefield NHS Trust, London, UK.

Correspondence: Brian G. Oliver, Woolcock Institute of Medical Research, Level 3 Cell Biology, 431 Glebe point road, Glebe, NSW, Australia 2037. E-mail: brian.oliver@uts.edu.au

Received: Oct 232018 | Accepted after revision: Jan 172019

Conflict of interest: None declared.

\section{References}

1 McNeill A, Brose L, Calder R, et al. E-cigarettes: an evidence update. A report commissioned by Public Health England. London, Public Health England, 2015.

2 Ween MP, Whittall JJ, Hamon R, et al. Phagocytosis and inflammation: exploring the effects of the components of E-cigarette vapor on macrophages. Physiol Rep 2017; 5: e13370.

3 Sussan TE, Gajghate S, Thimmulappa RK, et al. Exposure to electronic cigarettes impairs pulmonary anti-bacterial and anti-viral defenses in a mouse model. PLos One 2015; 10: e0116861.

4 Garcia-Arcos I, Geraghty P, Baumlin N, et al. Chronic electronic cigarette exposure in mice induces features of COPD in a nicotine-dependent manner. Thorax 2016; 71: 1119-1129.

5 Higham A, Rattray NJW, Dewhurst JA, et al. Electronic cigarette exposure triggers neutrophil inflammatory responses. Respir Res 2016; 17: 56.

6 Chen L, Ge Q, Tjin G, et al. Effects of cigarette smoke extract on human airway smooth muscle cells in COPD. Eur Respir J 2014; 44: 634-646.

7 Krimmer DI, Burgess JK, Wooi TK, et al. Matrix proteins from smoke-exposed fibroblasts are pro-proliferative. Am J Respir Cell Mol Biol 2012; 46: 34-39.

8 Kosmider L, Sobczak A, Fik M, et al. Carbonyl compounds in electronic cigarette vapors: effects of nicotine solvent and battery output voltage. Nicotine Tob Res 2014; 16: 1319-1326. 
9 Litt MD, Duffy V, Oncken C. Cigarette smoking and electronic cigarette vaping patterns as a function of e-cigarette flavourings. Tob Control 2016; 25: Suppl. 2, ii67-ii72.

10 Sharafkhaneh A, Hanania NA, Kim V. Pathogenesis of emphysema: from the bench to the bedside. Proc Am Thorac Soc 2008; 5: 475-477. 